\title{
Gadolinium Contrast Agents for CNS Imaging: Current Concepts and Clinical Evidence
}

E. Kanal, K. Maravilla, and H.A. Rowley

\begin{abstract}
SUMMARY: The aim of this article was to review the properties of the various gadolinium-based contrast agents used for CNS imaging along with the clinical evidence and published data that highlight the impact these different properties can have on diagnostic performance. In addition, approaches to optimizing image acquisition that take into account the different properties of specific gadoliniumbased contrast agents and an extensive review of the safety profiles of the various agents are presented.
\end{abstract}

ABBREVIATIONS: $\mathrm{CE}=$ contrast-enhanced; $\mathrm{CNR}=$ contrast-to-noise ratio; $\mathrm{GBCA}=$ gadolinium-based contrast agent; Gd = gadolinium; NSF = nephrogenic systemic fibrosis; rCBV = relative cerebral blood volume; $\mathrm{rCBF}=$ relative cerebral blood flow

O the 9 gadolinium-based contrast agents (GBCAs) approved by the United States FDA for contrast-enhanced (CE) MR imaging, 7 (gadoterate meglumine, Dotarem, Guerbet, Aulnay-sous-Bois, France; gadobutrol, Gadavist, Bayer HealthCare Pharmaceuticals, Wayne, New Jersey; gadopentetate dimeglumine, Magnevist, Bayer HealthCare; gadobenate dimeglumine, MultiHance, Bracco Diagnostics, Princeton, New Jersey; gadodiamide, Omniscan, GE Healthcare, Milwaukee, Wisconsin; gadoversetamide, OptiMARK, Covidien, Dublin, Ireland; gadoteridol, ProHance, Bracco Diagnostics) are approved specifically for CE-MR imaging of the CNS. ${ }^{1-7}$ The 2 agents not approved for CE-MR imaging of the CNS (gadofosveset trisodium, Ablavar, Lantheus Medical Imaging, North Billerica, Massachusetts; and gadoxetic acid, Bayer HealthCare Pharmaceuticals [Eovist or Primovist]) have distinct properties that render them unsuitable for this indication; Ablavar ${ }^{8}$ is an intravascular "blood-pool" agent approved for MR angiography of the aortoiliac vessels, whose strong binding to serum albumin (and resulting large effective molecular size) restricts permeability across the open blood-brain barrier, which limits suitability for CNS applications, while Eovist $^{9}$ is an approved liver-specific agent inappropriate for CNS

From Magnetic Resonance Services (E.K.), Department of Radiology, University of Pittsburgh Medical Center, Pittsburgh, Pennsylvania; Research Laboratory (K.M.), University of Washington, Seattle, Washington; and Departments of Radiology, Neurology, and Neurosurgery (H.A.R.), University of Wisconsin, Madison, Wisconsin.

Please address correspondence to Howard A. Rowley, MD, Department of Radiology, University of Wisconsin, 600 Highland Ave, Mail code 3252, Madison, WI 53792; e-mail: HRowley@UWHealth.org

- Indicates open access to non-subscribers at www.ajnr.org

三 Indicates article with supplemental on-line tables.

http://dx.doi.org/10.3174/ajnr.A3917 applications because $50 \%$ of the injected dose is taken up and eliminated by hepatocytes.

Although numerous studies published in high-ranking peerreviewed journals have confirmed the safety and efficacy of the 7 GBCAs approved for CNS imaging, differences among these agents and the impact these differences may have on diagnostic sensitivity and clinical decision-making remain underappreciated and sometimes misunderstood.

The aim of this article was to review the properties of the various GBCAs used for CNS imaging together with the clinical evidence and published data that highlight the impact these different properties can have on diagnostic performance. In addition, approaches to optimizing image acquisition that take into account the different properties of specific GBCAs and an extensive review of the safety profiles of the various agents will be presented.

For the purposes of the present review, brand names rather than chemical names have been used throughout to refer to the various GBCAs. Although chemical names would ordinarily be used in a review article such as this, it was thought that because the actual practitioners of MR imaging are typically more familiar with brand names than with chemical names, the use of brand names would help avoid the possibility of obfuscation and thereby enhance clarity in this field. Moreover, because generic formulations of these agents are not available in the United States, these brand names are clinically relevant designations. We have therefore elected to specifically use the same brand names for the various agents with which the reader will be most familiar.

\section{GBCAS: WHAT THEY ARE AND WHAT THEY DO}

As a heavy metal in the lanthanide series, elemental free gadolinium (Gd) is toxic to humans. GBCAs are formed by chelation of gadolinium to organic ligands to decrease its toxicity and render it 


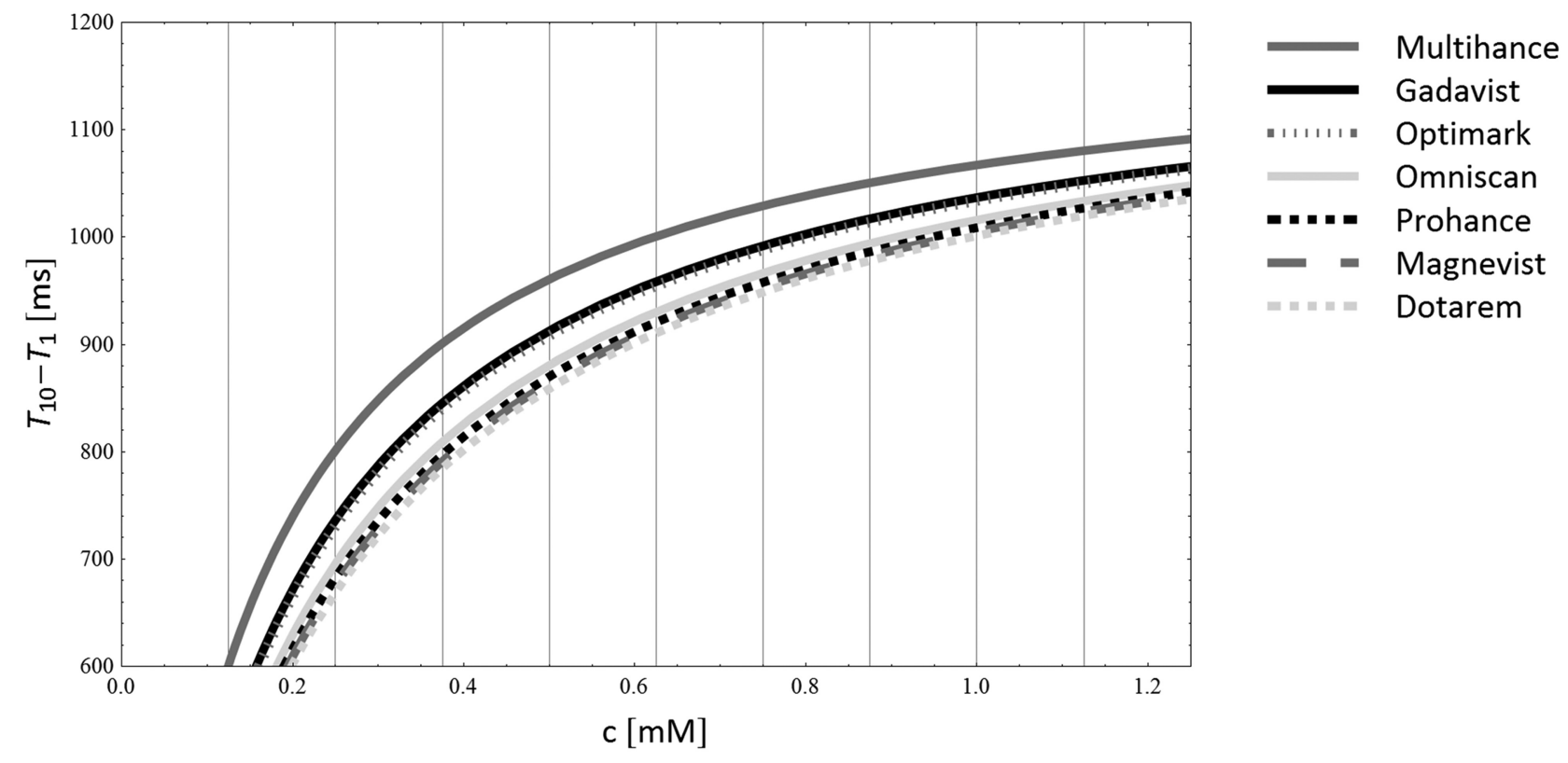

FIG 1. Comparison of $\mathrm{Tl}$ shortening of GBCAs as a function of $\mathrm{Gd}$ concentration, calculated from the rl-relaxivities in blood reported by Rohrer et al in 2005."

safe for human use. Structurally, there are 2 distinct categories of GBCAs: 1) macrocyclic molecules in which the $\mathrm{Gd}^{3+}$ ion is caged within the cavity of the ligand, and 2) open-chain or "linear" molecules (On-line Table 1). Within each category, there are both ionic and nonionic GBCAs. Despite their different molecular structures and physicochemical properties, all GBCAs used for CE-MR imaging of the CNS have an identical mechanism of action involving shortening of the $\mathrm{T} 1, \mathrm{~T} 2$, and $\mathrm{T} 2{ }^{*}$ relaxation time constants of adjacent water protons in tissues ${ }^{10}$ and similar biodistribution profiles because all are extracellular agents that do not cross the intact blood-brain barrier. The fundamental ability of GBCAs to selectively shorten T1 relaxation within a lesion compared with normal tissue is the basis for the most commonly applied usage of contrast agents in clinical practice today.

The extent to which the time constants are shortened, and hence the effectiveness of a specific GBCA in clinical practice, depends on the local concentration of the GBCA (Fig 1) and on the relaxivity values $r_{i}$ (with $\mathrm{i}=1,2,2^{*}$ ) of the agent. Relaxivities are defined as the slope of the linear regression generated from a plot of the measured relaxation rate $\left(1 / T_{\mathrm{i}}\right)$ versus the concentration of the GBCA:

$$
\frac{1}{T_{i}}=\frac{1}{T_{i(0)}}+r_{i} \times[\mathrm{GBCA}]
$$

where $T_{i}$ denotes the longitudinal (T1) or transverse (T2, $\mathrm{T} 2^{\star}$ ) relaxation times of a solution containing GBCA and $T_{i(o)}$, the relaxation time of the solvent without GBCA. ${ }^{11}$ Agents with higher $r 1, r 2$, and $r 2^{*}$ values will shorten the T1, T2, and T2* relaxation times of tissues to a greater extent, resulting in greater signal-intensity enhancement on T1-weighted images or greater signal-intensity loss on $\mathrm{T} 2$ - and $\mathrm{T} 2{ }^{*}$-weighted images, respectively.

Molecules normally tumble or rotate in space at a rate that is dependent on many factors, not the least of which is their molec- ular mass. This tumbling rate can also be represented as the inverse of the so-called molecular correlation time. The closer the tumbling rate of the gadolinium-containing chelate molecule to the resonant frequency of water molecules, the greater is the relaxivity or relaxation enhancement. The molecules of each of the 7 FDA-approved CNS gadolinium chelates are all relatively small and therefore have exceedingly high tumbling rates that exceed the resonant frequencies of water molecules at the clinically used MR imaging field strengths. However, as the molecular tumbling rates between the paramagnetic contrast agent and the target water-based molecules become closer, energy exchange between the 2 molecules is facilitated and longitudinal magnetization is enhanced (ie, the target tissue T1 shortens).

One of the approved CNS agents, MultiHance, interacts weakly and transiently with serum albumin in vivo. This interaction slows the molecular tumbling rate of the complex, rendering it closer to the resonant frequency of water molecules at the field strength at which they are being imaged. This increases the (enhanced) tissue rotational correlation time (ie, slows its tumbling rate) and therefore brings it closer to matching that of water. The result is greater relaxivities/r1 values and stronger relaxation enhancement effects. ${ }^{3,12,13}$ Thus, the $r 1$ of MultiHance measured in human plasma at $37^{\circ} \mathrm{C}$ has been reported to be as high as $7.9 \mathrm{~L} \times$ $\mathrm{mmol}^{-1} \times \mathrm{s}^{-1}$ at $1.5 \mathrm{~T},{ }^{14}$ whereas the reported $r 1$ values of GBCAs that do not significantly interact with serum albumin range from approximately $3.6 \mathrm{~L} \times \mathrm{mmol}^{-1} \times \mathrm{s}^{-1}$ at $1.5 \mathrm{~T}$ for Dotarem to $5.2 \mathrm{~L} \times \mathrm{mmol}^{-1} \times \mathrm{s}^{-1}$ at $1.5 \mathrm{~T}$ for Gadavist. ${ }^{11,14}$ However, reported relaxivity values may vary somewhat between investigating groups due to measurements being made under different conditions. For example, the $r 1$ and $r 2$ values reported by Rohrer et $\mathrm{al}^{11}$ were obtained by using nonsterile bovine blood plasma, while those reported by Pintaske et $\mathrm{al}^{14}$ were obtained by using human blood plasma.

Six of the 7 GBCAs approved for neuroradiologic imaging are 
formulated as $0.5 \mathrm{~mol} / \mathrm{L}$ concentrations, while 1 agent, Gadavist, is formulated at $1.0 \mathrm{~mol} / \mathrm{L}$. The physicochemical properties of this latter GBCA resemble those of the other conventional GBCAs, and its molecular structure differs from that of ProHance in that a hydroxypropyl group on the ProHance molecule is replaced by a trihydroxybutyl group on the Gadavist molecule. ${ }^{15}$ Thus, the slightly higher relaxivity of Gadavist can be ascribed mainly to the slightly larger size and hence slightly slower molecular tumbling rate of the Gadavist molecule itself. Notably, the magnitude of the slowing of the molecular tumbling rate is smaller than that observed for GBCAs approved for neuroradiologic applications that interact with serum albumin as well as non-neuroradiologic agents such as Ablavar for which powerful protein binding is observed.

The degree of tissue $\mathrm{T} 1$ (and T2 and $\mathrm{T} 2{ }^{*}$ ) shortening by any GBCA is determined by the local concentration of the agent within the tissue being imaged in combination with the relative relaxivity of the agent. Varied concentrations of administered GBCAs can be theorized to result in varied rates of uptake of the agent into the extracellular fluid of the tissues being imaged, with faster tissue uptake expected with a GBCA formulated at a greater concentration. Theoretically, albeit not demonstrated clinically, in routine T1-weighted steady-state imaging, the higher concentration of Gadavist might impact the "ideal" postcontrast imaging time for this agent versus others with lower molarity. Recalling, however, that intravenous administration requires that all administered boluses pass through the heart and lungs before being distributed to the rest of the body, it would seem that any concentration difference per time among the various neuroradiologic GBCAs, other than possibly first-pass effects, would be quite small and likely not clinically discernable in steady-state. In addition, one should recognize that even if the contrast agent may theoretically accumulate faster in the target lesion, enhancing background tissues would also be expected to similarly accumulate that same amount more rapidly. Notwithstanding these theoretic considerations, in daily clinical practice, it appears that the ultimate steady-state concentrations in the tissues being imaged are sufficiently similar for all GBCAs regardless of the administered concentration and that the final concentration in tissues in steady-state is most likely determined predominantly by the total administered dose, based on the similarity of observed contrast enhancement when corrected for differences in relative relaxivity of the different agents as discussed later in this review. Thus, for routine, equidose, postcontrast T1-weighted steady-state imaging, enhanced signal intensities are dominated predominantly by the relative relaxivity values of the agents being compared. Therefore, the higher the $r 1$ value, the greater will be the contrast between the lesion and nonenhancing background tissues.

Dynamic phase imaging (as is used in perfusion-weighted imaging and in contrast-enhanced MR angiographic studies) differs from the above in that the target tissue of interest is the bloodthe same "tissue" into which the agent is directly injected. Thus, per the equation above, higher GBCA concentration and/or higher relaxivity could each produce increases in signal intensity of the intravascular blood for T1-weighted imaging, or greater signal loss for T2- or T2*-weighted imaging. For dynamic bolus CE-MRA examinations, a principal objective is to time the bolus arrival within the vessels of interest to coincide with acquisition of the lowest order phase-encoding steps in $k$-space to obtain maximum vessel enhancement. Because all 7 GBCAs approved for neuroradiologic imaging are approved at identical administered total doses of $0.1 \mathrm{mmol} / \mathrm{kg}$ body weight, this means that the higher the concentration of the administered agent, the smaller the volume injected. Thus, a standard administered dose to a 70-kg patient would be $14 \mathrm{~mL}$ of any of the 6 GBCAs formulated at 0.5 $\mathrm{mol} / \mathrm{L}$, while it would be $7 \mathrm{~mL}$ for Gadavist formulated at 1.0 $\mathrm{mol} / \mathrm{L}$. However, the result of a smaller injected volume is that the timing window of opportunity during which to catch the higher concentration Gadavist in the vessels of interest is shortened by as much as one-half, making bolus timing more difficult and potentially more error-prone. This can possibly be offset by halving the injection rate when administering higher concentration agents or by diluting them with normal saline before administration. However, these approaches would negate any theoretic advantage of using a higher concentration agent.

The relatively recent FDA approval of GBCAs formulated at different concentrations but approved at identical administered doses provides opportunities for comparison among such agents. However, such attempts at comparison should use identical FDA-approved doses; comparisons should not be performed by using arbitrarily defined equivalent volumes because this would result in "double-dosing" of the higher concentration agent. Such studies evaluating agents of different concentrations would be useful because the ideal time to image is dependent on many factors, which include blood vessel size, flow rate and transit time in the case of CE-MRA, perfusion dynamics (vascularity and permeability) of the anatomy/ pathology to be imaged in the case of perfusion MR, the concentration and relaxivities of the administered agent, and the design of the MR imaging pulse sequence to be used to acquire the postcontrast images. Furthermore, "ideal" timing will also be highly dependent on whether first-pass, dynamic contrast information is sought as opposed to steady-state images. "Ideal" timing tends to be spread over far longer windows of opportunity for steady-state acquisitions, while the window for first-pass, dynamic contrast information is quite short and is far more significantly impacted by such factors as the manner in which $k$-space is filled and the temporal resolution of the acquired images. Imaging parameters and timing that might be ideal for one agent might prove to be quite different for another GBCA with different concentration or relaxivity.

\section{CLINICAL EVIDENCE FOR THE ROLE OF RELAXIVITY ON DIAGNOSTIC PERFORMANCE Methodologic Considerations}

Early studies to compare contrast agents for diagnostic efficacy were designed exclusively as interindividual parallel group studies in which each patient (with varying disease entities) was randomly assigned to receive just 1 of the 2 contrast agents. ${ }^{16-25}$ Unfortunately, studies of this type are subject to wide interpatient and interlesion variability, resulting in disparate variations within each arm of the study. Such studies do not permit reliable demonstrations of relevant differences between study groups and agents. Indeed, such parallel group studies can only demonstrate equivalence between study groups for equivalent doses of different GBCAs and not superiority or inferiority, even among GBCAs with widely varying relaxivity values. $^{24,25}$

AJNR Am J Neuroradiol 35:2215-26 Dec 2014 www.ajnr.org 2217 
Direct comparison among different agents is accomplished more objectively with an intraindividual crossover study in which each patient receives both GBCAs in random order in 2 identical MR imaging examinations separated by just a few days. Such studies are better designed to isolate the GBCA as the only variable being assessed. Thus, variations due to patient-, disease-, or examination-related factors are eliminated or, at least, minimized. In such studies, any differences in findings can be attributed solely to the GBCA because all other variables are identical for the 2 examinations.

Summarized below are the findings of several such intraindividual crossover studies performed in human subjects for CNS evaluation of various GBCAs. In organizing this discussion, we realized that there is no standardized definition for use of the term, "high relaxivity." It is the opinion of these authors that "high relaxivity" should be defined not numerically alone, but rather by an objectively proved ability of an agent to deliver increased clinical utility as measured by clinically relevant increases in signal enhancement (rather than merely small but statistically significant signal increases) or, preferably, an objectively measured increase in lesion number or lesion extent compared with other "standard" GBCAs.

Steady-State Imaging. To analyze the possible differential roles of relaxivity and concentration in imaging performance, findings are presented for studies that compared the following: 1) Dotarem, Magnevist, Omniscan, OptiMARK, and ProHance (which all possess similar [standard] relaxivity values and are formulated at standard $[0.5 \mathrm{~mol} / \mathrm{L}]$ concentrations), 2) Gadavist (the GBCA with slightly higher relaxivity and the highest concentration [1 $\mathrm{mol} / \mathrm{L}]$ ), and 3) MultiHance (the GBCA with the highest relaxivity and formulated at standard concentration).

1) Intraindividual Crossover Studies Comparing GBCAs with Standard Relaxivity/Standard Concentration (Dotarem, Magnevist, Omniscan, OptiMARK, ProHance). To the authors' knowledge, Greco et $\mathrm{al}^{26}$ performed the only intraindividual crossover study that compared GBCAs with standard relaxivity/standard concentration. In that study, 2 blinded readers intraindividually compared Magnevist (r1-relaxivity at 1.5T: 3.9-4.1 $\mathrm{L} \times \mathrm{mmol}^{-1} \times$ $\left.\mathrm{s}^{-1}\right)^{11,14}$ with ProHance ( $r$-relaxivity at $1.5 \mathrm{~T}: 4.1 \mathrm{~L} \times \mathrm{mmol}^{-1} \times$ $\mathrm{s}^{-1}$ measured in bovine plasma at $\left.37^{\circ} \mathrm{C}\right)^{11}$ in 80 subjects for the presence of disease, degree of enhancement, location and number of lesions, and additional information gained (definition of lesion borders, improved visualization, distinction of edema, disease classification, determination of recurrent tumor, and so forth). Neither reader noted any significant differences in terms of GBCA preference (readers 1 and 2 preferred ProHance over Magnevist in 2 and 4 cases, respectively, and Magnevist over ProHance in 1 and 2 cases, respectively), and no differences were noted between agents in terms of the additional information provided on postcontrast images (On-line Table 2).

2) Intraindividual Crossover Studies Comparing a GBCA with Slightly Higher Relaxivity and High Concentration versus Standard Relaxivity/Standard Concentration Agents (Gadavist versus Either Dotarem, Magnevist, Omniscan, OptiMARK, or ProHance). To date, 5 published reports have described intraindividual crossover comparisons of Gadavist with standard relaxivity GBCAs. ${ }^{27-31}$ Three of these looked specifically at the potential benefit of Gadavist versus Magnevist ${ }^{27,28}$ or ProHance ${ }^{29}$ for the detection and visualization of cerebral metastases and concluded, in each case, that Gadavist is advantageous for lesion detection primarily because of improved lesion conspicuity (On-line Table 2). However, the conclusions in a study by Anzalone et $\mathrm{al}^{27}$ were based solely on subjective assessment of images from 27 patients by 2 neuroradiologists in consensus, but unfortunately, no quantitative assessment of lesion enhancement was reported. Kim et $\mathrm{al}^{28}$ reported improved quantitative enhancement (lesion/brain contrast-tonoise ratio $[\mathrm{CNR}]$ ) with Gadavist, but this study was retrospective and only compared double $(0.2 \mathrm{mmol} / \mathrm{kg}$ body weight $)$ doses of Gadavist and Magnevist. Furthermore, GBCA administration was not random (all patients received Magnevist for the first examination and Gadavist for the second). In the third study in patients with brain metastases, Katakami et $\mathrm{al}^{29}$ evaluated a larger number of patients by using a prospective design and concluded that a single $0.1-\mathrm{mmol} / \mathrm{kg}$ dose of Gadavist is noninferior to a double $0.2-\mathrm{mmol} / \mathrm{kg}$ dose of ProHance for lesion detection. However, despite administering a single $0.1-\mathrm{mmol} / \mathrm{kg}$ dose of ProHance as part of the study design, no assessment of single-dose ProHance images was performed. Thus, it is not possible to say whether a single dose of ProHance would have proved noninferior to a single dose of Gadavist by using their study design, sample size, and assessment methodology.

In a more recent single-center study in 51 patients with either primary or secondary brain tumors, 2 blinded readers each preferred Gadavist to ProHance in more patients in terms of subjective "preference in contrast enhancement," "overall preference," and "preference in diagnostic quality." ${ }^{30}$ However, differences in quantitative enhancement were inconsistent and sequence-dependent, with a higher SNR for Gadavist noted only on a second T1-FLASH sequence at approximately 10 minutes postinjection, with no differences between agents seen on T1-spin echo or MPRAGE sequences. Indeed, Bayer HealthCare (manufacturer of gadobutrol, Gadavist) reported to the FDA that the performances of 0.1-mmol/ $\mathrm{kg}$ doses of Gadavist and ProHance for brain tumor imaging are similar. ${ }^{2,31}$ In a prospective multicenter phase III study performed in 419 patients for the FDA approval of Gadavist for CNS imaging, 3 blinded readers each reported similar contrast enhancement, lesion border delineation, and lesion internal morphology and a similar overall accuracy of diagnosis when these 2 agents were administered at an equivalent dose of $0.1 \mathrm{mmol} / \mathrm{kg}$ body weight. The conclusion of the study was that Gadavist is noninferior to ProHance. ${ }^{2,31}$

Another recent report presented findings from a study that prospectively compared single-dose Gadavist (r1-relaxivity: 4.7$5.2 \mathrm{~L} \times \mathrm{mmol}^{-1} \times \mathrm{s}^{-1}$ in plasma at $37^{\circ} \mathrm{C}^{11,14}$ ) with single-dose Dotarem ( $r$ l-relaxivity: $3.6 \mathrm{~L} \times \mathrm{mmol}^{-1} \times \mathrm{s}^{-1}$ ) in 136 patients with cerebral neoplastic enhancing lesions. ${ }^{32}$ In this study, significant preference for Gadavist compared with Dotarem was noted by 2 of 3 blinded readers for overall reader preference. However, none of the 3 readers considered Gadavist superior to Dotarem for lesion delineation, while only 1 blinded reader noted a minimally significant preference for Gadavist for the definition of lesion internal structure. Quantitatively, the percentage lesion enhancement following Gadavist was approximately $9 \%$ higher than 
that following Dotarem, as expected from the differences in their respective relaxivities, but this yielded no significant difference between the 2 agents for measured CNR. Most important, no differences in the number of lesions detected with either agent were observed.

3a) Intraindividual Crossover Studies Comparing the GBCA with the Highest Relaxivity/Standard Concentration versus GBCAs with Standard Relaxivity/Standard Concentration (MultiHance versus Dotarem, Magnevist, Omniscan, OptiMARK, or ProHance). Numerous multicenter studies have compared MultiHance with standard GBCAs by using an intraindividual crossover study design with blinded image evaluation by fully independent experienced neuroradiologists. ${ }^{33-41}$ All of these studies were designed to demonstrate superiority rather than noninferiority, and the findings of all concluded that MultiHance is significantly superior in terms of both qualitative enhancement (global diagnostic preference, lesion border delineation, definition of disease extent, visualization of lesion internal morphology, lesion contrast enhancement) and quantitative enhancement (CNR, lesion-tobackground ratio) (On-line Table 2). In each of these studies, the authors concluded that the superiority of MultiHance was due to its higher $r 1$ value.

3b) Intraindividual Crossover Study to Compare the Highest Relaxivity GBCA versus the GBCA Formulated at the Highest Concentration (MultiHance versus Gadavist). A recent study by Seidl et $\mathrm{al}^{41}$ directly addressed the relative merits of high relaxivity versus high gadolinium concentration. In their randomized, doubleblind, intraindividual crossover study, 123 patients each underwent 1 examination with $0.1-\mathrm{mmol} / \mathrm{kg}$ MultiHance and 1 examination with $0.1-\mathrm{mmol} / \mathrm{kg}$ Gadavist. Three blinded readers consistently demonstrated a highly significant $(P<.0001)$ preference for MultiHance for all qualitative end points with good interreader agreement for all evaluations (On-line Table 2). In addition, significant superiority was noted for all quantitative assessments with a mean difference of approximately $22 \%$ in percentage lesion enhancement between MultiHance and Gadavist.

This study demonstrated that gadolinium concentration has little-to-no practical clinical impact on steady-state morphologic imaging and that at identical approved $(0.1 \mathrm{mmol} / \mathrm{kg})$ doses, the relaxivity of the GBCA is the dominant characteristic determining the degree of enhancement.

Perfusion Imaging. Cerebral perfusion assessment by dynamic susceptibility contrast MR imaging is frequently used for evaluation of brain tumors, stroke, and degenerative diseases such as dementia. The technique is based on rapid intravenous injection of a GBCA and subsequent bolus tracking by using a fast susceptibility-weighted imaging sequence that uses the $\mathrm{T} 2^{*}$ relaxing properties of the GBCA. Following tracer kinetic modeling, parametric maps of mean transit time, regional cerebral blood volume (rCBV), and regional cerebral blood flow (rCBF) can be calculated by unfolding tissue concentration curves and the concentration curve of the feeding artery.

Compared with conventional morphologic (static) imaging, (dynamic) perfusion imaging is more dependent on the shape of the injected contrast bolus and thus on the rate at which GBCAs are injected. Additionally, higher administered GBCA concentra- tion and higher relaxivity might each be beneficial in augmenting the signal loss associated with the first-pass contrast bolus through the tissues of interest.

Although interindividual parallel group studies have compared Gadavist with Magnevist at $1.5 \mathrm{~T},{ }^{42,43}$ comparatively few intraindividual crossover studies have been performed to compare GBCAs for perfusion imaging. Those that have been performed have compared Gadavist with either Magnevist at $3 \mathrm{~T}^{44}$ or MultiHance at $1.5 \mathrm{~T}^{45}$ or $3 \mathrm{~T} .^{46,47}$

1) Inter- and Intraindividual Crossover Studies Comparing GBCAs with Standard Relaxivity/High Concentration versus Standard Relaxivity/Standard Concentration (Gadavist versus Magnevist). An interindividual parallel group comparison of Gadavist with Magnevist was first performed by Griffiths et al, ${ }^{42}$ who compared 10and 20 -mL injections of Gadavist with 20 -mL injections of Magnevist (all at $5 \mathrm{~mL} / \mathrm{s}$, resulting in overall injection times of 2 and 4 seconds, respectively) in 6 groups of 6 patients ( 36 patients overall) at $1.5 \mathrm{~T}$ to determine whether the higher Gd concentration of Gadavist was beneficial when using thinner sections $(4 \mathrm{~mm}$ as opposed to $7 \mathrm{~mm}$ ) for single-shot, gradient-recalled echo-planar imaging. They compared time-intensity curves calculated at regions of interest in the hemispheric white matter and thalamus in terms of maximum signal reduction (ie, the difference between mean baseline and minimum value on the time-intensity curve), full width at half minimum, and signal-to-noise measurements. No significant differences were found between $20 \mathrm{~mL}$ of Magnevist and $10 \mathrm{~mL}$ of Gadavist in terms of the maximum signal changes observed in either anatomic area and at either section thickness. On the other hand, the signal changes nearly doubled when 20-mL Gadavist was compared with 20-mL Magnevist (ie, when a 2-fold higher dose of Gadavist was used), indicating that the total amount (ie, dose) of Gd was the dominant factor in determining signal response rather than the administered concentration per se.

A second interindividual parallel group comparison of Gadavist and Magnevist at $1.5 \mathrm{~T}$ was subsequently performed by the same group when investigating whether 2 gadolinium perfusion studies of the whole brain could be performed during the same table occupancy without degradation of the derived data in the second study. ${ }^{43}$ In this study, 12 patients each received 2 injections at a fixed rate of $5 \mathrm{~mL} / \mathrm{s}$ of either 20-mL Magnevist (6 patients) or 10 -mL Gadavist (6 patients), with each administration separated by 8 minutes. Although the study was not designed specifically to compare the 2 agents directly, the authors nevertheless showed no significant differences in either the maximum signal change or full width at half maximum with 10 -mL Gadavist compared with 20-mL Magnevist.

A small-scale intraindividual crossover comparison of these 2 GBCAs was recently performed at $3 \mathrm{~T}$ by Giesel et $\mathrm{al}^{44}$ in 11 patients ( 6 with intra-axial tumors, 5 with extra-axial tumors), who each underwent examinations with $5-\mathrm{mL}$ Gadavist and $10-\mathrm{mL}$ Magnevist by using a T2*-weighted, gradient recalled-echo, echoplanar technique. As in the studies by Griffiths et al, ${ }^{42,43}$ the injection rate for both agents was $5 \mathrm{~mL} / \mathrm{s}$. However, unlike Griffiths et al, the authors reported significantly higher maximal signal changes for Gadavist in both gray and white matter and noted that Gadavist depicted a larger number of "hot spots" (areas with higher blood perfusion in the tumor) on color-coded maps than 
Magnevist in most of the 6 intra-axial tumors. The authors concluded that the higher concentration of Gadavist offers advantages over standard-concentration Magnevist for delineation of gray and white matter and for the demarcation of highly vascularized tumor tissue and that these advantages are due to an improved bolus effect with increased intravascular concentration during the first pass.

2) Intraindividual Crossover Studies Comparing High Relaxivity/ Standard Concentration versus Standard Relaxivity/High Concentration (MultiHance versus Gadavist). Early intraindividual crossover studies to compare Gadavist and MultiHance were performed independently by Essig et $\mathrm{al}^{45}$ and Thilmann et $\mathrm{al}^{46}$ in healthy volunteers at $1.5 \mathrm{~T}$ and $3 \mathrm{~T}$, respectively. In the study by Thilmann et al, ${ }^{46} 16$ healthy volunteers each underwent 3 DSC-MR imaging examinations separated by at least 3 days, receiving a single $(0.1-\mathrm{mmol} / \mathrm{kg} ; 7-\mathrm{mL})$ dose of Gadavist, a double (14-mL) dose of Gadavist, and a single (14-mL) dose of MultiHance, each at an injection rate of $5 \mathrm{~mL} / \mathrm{s}$ (ie, resulting in injection times of 1.4, 2.8, and 2.8 seconds, respectively). Quantitative determinations based on signal intensity/time curves were made at regions of interest on gray and white matter and specific arteries selected for perfusion analysis. Additionally, gray-scale and colorcoded maps of regional cerebral blood volume and regional cerebral blood flow were calculated and compared.

Quantitative analysis revealed nearly identical signal intensity/ time curves for the 2 single-dose examinations. No differences were noted in terms of maximal relative signal drop, full width at half maximum, or signal-to-noise ratio of the concentration curve at maximum concentration. Likewise, qualitative evaluation of rCBV and rCBF maps by 2 experienced blinded radiologists revealed no differences between the 2 single-dose examinations with no advantage noted for either of the 2 GBCAs. More pronounced signal drops (52\% versus 32\%) and better quality perfusion maps ( $\mathrm{rCBV}$ and $\mathrm{rCBF}$ ) were obtained with doubledose Gadavist compared with either single-dose examination, though both single-dose examinations were considered suitable for diagnostic purposes.

More recently, Wirestam et $\mathrm{al}^{47}$ performed further evaluations of data acquired by Thilmann et $\mathrm{al}^{46}$ and confirmed that doubledose Gadavist results in higher absolute CBV, CBF, and mean transit time than single-dose Gadavist and that no significant differences are apparent between single-dose Gadavist and singledose MultiHance.

Similar findings and conclusions to those of Thilmann et $\mathrm{al}^{46}$ were made by Essig et $\mathrm{al}^{45}$ in a study comprising 12 healthy male volunteers who each underwent 4 highly standardized perfusion MR imaging examinations with 0.1 - and $0.2-\mathrm{mmol} / \mathrm{kg}$ doses of Gadavist and MultiHance, each administered at $5 \mathrm{~mL} / \mathrm{s}$. As in the study by Thilmann et al, ${ }^{46}$ a single dose of both agents was shown to be sufficient to achieve high-quality, diagnostically valid perfusion maps. Again, no differences were noted between single doses of the 2 agents for any quantitative parameter (susceptibility effect [percentage signal drops of approximately 30\%], rCBV, and $\mathrm{rCBF}$ values) apart from full width at half maximum, which was significantly greater for MultiHance. Likewise, 2 off-site blinded readers found no significant differences between Gadavist and MultiHance in terms of image quality, adequacy of white-to-gray matter differentiation, or subjective preference for 1 agent or the other in terms of CBV and CBF image sets. Better overall image quality was noted with double $(0.2 \mathrm{mmol} / \mathrm{kg})$ doses of the 2 agents, for which a slightly higher susceptibility effect was seen with Gadavist. Nevertheless, the authors considered that double doses of the 2 agents provided no clinical benefit over single-dose examinations. The authors also concluded that single doses of both agents were effective at inducing sufficient signal drop on $\mathrm{T} 2{ }^{\star} \mathrm{EPI}$ sequences to permit robust and reproducible quantification of perfusion parameters. Moreover, they concluded that the greater volume of injection of MultiHance had no disadvantage and gave comparable perfusion values to those obtained with the more highly concentrated Gadavist.

Contrast-Enhanced MR Angiography. Similar to perfusion imaging, dynamic bolus contrast-enhanced MR angiography is a rapid imaging technique in which images are acquired during the first pass of a GBCA through the vessels of interest. However, unlike DSC perfusion imaging, the level of enhancement is dependent on the $r 1$ of the agent rather than the $r 2^{*}$ value. Accordingly, image quality and diagnostic performance are dependent not only on the image acquisition parameters but also on the contrast-injection protocol. Thus, while advances in sequence design can lead to marked improvements in the spatial and temporal resolution of vessel images, it remains fundamental that bolus timing and the peak concentration of intraluminal contrast coincide with the acquisition of the lower order phase-encoding steps of $k$-space image acquisition. To achieve MR angiograms with adequate homogeneous arterial contrast and without image artifacts, contrast bolus timing must achieve high concentration and a relatively constant plateau during acquisition of the central part of $k$-space, which contributes most of the image contrast. In addition, evidence appears to support the need to maintain a high level of Gd in the vessels during much of the higher order phase-encoding acquisition to minimize vessel edge blurring that can reduce vessel detail and visualization of smaller vessels. ${ }^{48}$

For the purposes of the present article, the focus will mainly be on studies comparing GBCAs for CE-MRA of the intracranial and supra-aortic vessels. However, the underlying principles of GBCA administration and image acquisition are common to all CEMRA examinations across all vascular territories.

\section{Intraindividual Crossover Studies Comparing GBCAs for CE-MRA of the Supra-Aortic Vessels}

Of the few intraindividual crossover studies performed in the supra-aortic vessels, most have compared MultiHance with Magnevist. ${ }^{49-51}$ In a very early study of 12 patients referred for CE-MRA of the carotid arteries, Pediconi et $\mathrm{al}^{49}$ compared a single 0.1 $\mathrm{mmol} / \mathrm{kg}$ dose of MultiHance with a double $(0.2-\mathrm{mmol} / \mathrm{kg})$ dose of Magnevist and found superior quantitative and qualitative enhancement with MultiHance for carotid time-resolved CE-MRA. Both doses of GBCAs were administered at a fixed rate of $2 \mathrm{~mL} / \mathrm{s}$, and the better imaging performance was ascribed to the higher $r 1$ of MultiHance. In that study, a single $0.1-\mathrm{mmol} / \mathrm{kg}$ dose of MultiHance would have been administered during 7.5 seconds for a $75-\mathrm{kg}$ patient. Conversely, the double $0.2-\mathrm{mmol} / \mathrm{kg}$ dose of Magnevist would have been administered during 15 seconds, potentially resulting in exclusion of a portion of the increased Magn- 
evist dose from the central part of the $k$-space during the MRA acquisition. On the other hand, the extended injection time for double-dose Magnevist would have provided double the window of opportunity to correctly "catch" the highest intraluminal GBCA concentration and may have contributed to better vessel wall sharpness, though this was not evaluated in the study. Empiric adjustments to optimize signal by using a specific pulse sequence, gadolinium agent, acquisition timing, and injection parameters are, therefore, critical in achieving best image quality.

A recent study by Li et $\mathrm{al}^{50}$ in 46 patients compared single-dose MultiHance and double-dose Magnevist. In this study, the 2-fold greater volume of Magnevist required to achieve a double dose was injected at a 2-fold faster rate to achieve comparable bolus geometry for the 2 examinations in each patient. Three blinded readers in the study found no differences between single-dose MultiHance and double-dose Magnevist for any qualitative parameter (vessel anatomic delineation, detection/exclusion of pathology, and global preference) or for quantitative measures of contrast enhancement (SNR, CNR). Indirect support for the findings of $\mathrm{Li}$ et $\mathrm{al}^{50}$ comes from a study by Bültmann et $\mathrm{al},{ }^{51}$ who compared single 0.1-mmol/kg doses of MultiHance and Magnevist across 19 arteries/arterial segments (comprising the internal carotid arteries; anterior, middle, and posterior cerebral arteries; vertebral arteries; and basilar artery) in 12 healthy volunteers at 3T. Maximum-intensity-projection images acquired with MultiHance were found to be markedly superior in terms of mean technical quality and vessel delineation to those acquired with Magnevist. Likewise the relative CNR was significantly greater with MultiHance, with overall increases of $23.3 \%, 26.7 \%$, and $28.5 \%$ noted for the internal carotid, middle cerebral, and basilar arteries, respectively.

More recently, Kramer et $\mathrm{al}^{52}$ compared Gadavist with both MultiHance and Dotarem in 20 healthy volunteers at 3T. Although the total dose of each GBCA administered was 0.1 $\mathrm{mmol} / \mathrm{kg}$ body weight, at variance with previous studies, the authors acquired both static CE-MRA and dynamic (time-resolved) CE-MRA images with $0.07 \mathrm{mmol} / \mathrm{kg}$ injected initially for the acquisition of static images followed by a further $0.03 \mathrm{mmol} / \mathrm{kg}$ for the acquisition of dynamic MRA. A fixed injection rate of $2 \mathrm{~mL} / \mathrm{s}$ was used for both injections with all 3 GBCAs, and determinations were made of both quantitative and qualitative end points. Qualitative assessment of static images by 3 blinded readers found Gadavist to be not significantly different from MultiHance but superior to Dotarem, while few differences were noted between MultiHance and Dotarem. In terms of quantitative assessment of static images, a higher SNR with Gadavist was noted in the proximal ICA but not in the distal ICA, while the CNR with Gadavist was not significantly different from that with MultiHance but significantly superior to that with Dotarem. Similar findings were obtained for dynamic MRA. Finally, no differences were noted between the different GBCAs in terms of vessel sharpness.

The manner in which the contrast agents were administered for this study is not one routinely used in clinical practice. Nevertheless, it supports the advantages potentially gained with increased GBCA concentration if data acquisition can be appropriately timed to the shortened first pass of contrast bolus.

\section{SAFETY}

With recognition of the association between Gd and nephrogenic systemic fibrosis (NSF) in 2006, there has been a sharply increased focus on GBCA safety. The 7 GBCAs currently approved by the FDA for CNS imaging have nearly identical pharmacokinetic (biodistribution and blood half-lives) profiles and mechanisms of action, and 6 of the 7 have similar clearance pathways almost exclusively through the kidneys. MultiHance differs in that a small fraction of the injected dose (approximately 3\%-5\%) is taken up by normally functioning hepatocytes and excreted into the bile.

\section{Nephrogenic Systemic Fibrosis}

NSF is a rare, potentially life-threatening disease that has been linked to the administration of some GBCAs in patients with severe renal impairment. In the peer-reviewed literature, approximately $78 \%$ of all unconfounded, single-agent cases of NSF have been associated with Omniscan, while a further $20 \%$ have been associated with Magnevist, and $<2 \%$, with OptiMARK. ${ }^{53}$ Very few single-agent cases $(0.5 \%)$ have been associated with the macrocyclic agent Gadavist, while no unconfounded cases have been reported for Dotarem, MultiHance, or ProHance. ${ }^{53}$ Similarly, no unconfounded cases have been reported for the 2 GBCAs not approved for CNS imaging (Ablavar, Eovist). Of note, GBCAs associated with the lowest number of putative cases of NSF, if any, are characterized either by a macrocyclic structure with high kinetic stability (Dotarem, Gadavist, ProHance) or a unique ability to interact with or bind to plasma proteins (Ablavar, Eovist, MultiHance). With the latter group, it has been proposed that the aromatic moiety on these complexes, in addition to being responsible for the protein-binding characteristics, may also increase the stability of the molecule by improving their kinetic inertia. This is possibly due to the steric effect of the bulky substituents that slightly hinders unwrapping of the ligand around the gadolinium. ${ }^{54}$

The introduction of specific recommendations such as patient prescreening for renal disease, the contraindication of less stable so-called high-risk GBCAs (Magnevist, Omniscan, and OptiMARK) in patients at risk of NSF, restricting the use of GBCAs to the lowest dose needed to provide the required diagnostic information, and a drastic reduction in the number of cases in which the recommended single dose is exceeded, has resulted in a markedly reduced incidence of NSF. Indeed, these measures have resulted in the number of new NSF cases dropping close to zero. ${ }^{55}$

\section{Adverse Reactions}

Although the tolerability of GBCAs is very good, adverse reactions are observed after the administration of all agents as reported in the prescribing information approved by regulatory authorities. On the basis of published data, rates of adverse reactions following GBCA administration range between $0.03 \%$ and $2.4 \% .^{56-65}$ Of these reactions, $>74 \%$ are transient and mild in intensity. Reactions of moderate or severe intensity have been reported to range from $<1 \%$ to $19 \%$ of cases. ${ }^{56,58,61-66}$ Life-threatening and fatal reactions are very rare, with 40 deaths per 51 million administered GBCA doses reported between 2004 and 2009. ${ }^{65}$

Adverse drug reactions fall into 2 major categories. The first 
includes reactions that can occur in any patient, such as drug overdose and drug interactions; the second includes reactions that are restricted to susceptible patients, such as drug toxicity/augmented effects (ie, drug idiosyncrasy or reduced tolerance) and hypersensitivity reactions that are either allergic or pseudoallergic in nature. ${ }^{67}$ These latter 2 reactions are clinically indistinguishable because their symptomatic presentations are very similar; however, they differ vastly mechanistically. Although true allergic reactions to GBCAs have been reported, ${ }^{68,69}$ the available evidence suggests that most adverse reactions to GBCAs are pseudoallergic in nature and are not associated with immunologic specificity. ${ }^{57}$

Acute reactions typically affect susceptible patients and occur within the first hour after GBCA injection. Mild reactions, such as nausea and vomiting, are usually self-limiting and do not require treatment. ${ }^{57,70}$ Patients who experience mild reactions should be monitored for at least 30 minutes post-GBCA exposure to ensure that there is no progression of signs and symptoms. ${ }^{57} \mathrm{Mod}-$ erate reactions consist of more severe symptoms than mild reactions, or they can appear as other clinically significant reactions that require specific treatment. The vital signs of patients suspected of experiencing a moderate reaction should be monitored closely to make certain that progression to a severe event is avoided. Severe reactions, though quite rare, are significant in that they can present life-threatening situations; thus, immediate recognition and treatment are warranted. In many cases, lifethreatening events begin with mild signs and symptoms, and then evolve rapidly. As a result, it is imperative that practitioners monitor all patients receiving a GBCA during their MR imaging procedure, with particular attention paid to asthmatic and atopic patients and patients with allergic respiratory phenomena because these patients are considered more "at risk" of an adverse event. Recent legal action and findings confirm the seriousness with which the Centers for Medicare and Medicaid Services approaches the mandate that GBCA administration be under direct physician supervision. ${ }^{71}$

Regulatory authorities have not differentiated among commercially available GBCAs in terms of acute adverse-reaction profiles. Most published reports consider GBCAs to have comparable adverse event profiles for mild reactions such as nausea and for severe anaphylactoid reactions. ${ }^{72}$ To remain objective and scientifically sound, comparisons of adverse event rates across agents should ensure that the mode of data collection (study design, prospective versus retrospective) and population (sex, clinical profile, body area) are taken into account. Variations in these factors alone can result in up to 100 -fold differences in apparent event rates. ${ }^{63-65,73-75}$ When differences in incidence rates among GBCAs are encountered, such differences are sometimes attributable to methodologic bias or to the so-called Weber or Lalli effects. $^{76,77}$

The Weber effect occurs when a new medication introduced to the clinical market leads to heightened safety vigilance on the part of practitioners and, as a consequence, more frequent reporting of adverse reactions. ${ }^{56,76,78}$ After experience is accumulated by using a given product, previously noteworthy reactions are perceived as being predictable and less noteworthy and, therefore, may be reported less frequently. ${ }^{56,76,78}$ Thus, the Weber effect is character- ized by a transient increase in adverse event reporting that tends to peak in the second year following introduction of a new agent, with a subsequent return to the original baseline reaction rate observed before the introduction of the new agent. ${ }^{76}$ Such an effect was recently reported with GBCAs by Davenport et $\mathrm{al}^{79}$ who described the impact of an abrupt switch from Magnevist to MultiHance on the incidence of immediate allergic-type adverse events. After MultiHance was substituted for Magnevist, a significant transient increase in the frequency of reported allergic-like reactions occurred that peaked in the second year postswitch and then declined, suggestive of the Weber effect. The subsequent reaction rate during last 3 calendar quarters of the monitoring period did not differ significantly from the original baseline reaction rate with Magnevist. ${ }^{79}$ However, the authors did note that the study was underpowered to confirm noninferiority among different GBCAs with respect to severe reactions.

Another confounder in the comparison of adverse events among various GBCAs is the Lalli effect, which occurs when practitioners unintentionally project their feelings about a new product onto patients, inducing fear and anxiety. This may result in unpredictable, idiosyncratic reactions of varying severity. ${ }^{77}$

Both of these effects have been observed with contrast agents ${ }^{56,77,79}$; however, true differences between agents in terms of severe acute reaction rates have not been demonstrated in wellcontrolled, statistically powered, prospective, randomized, blinded studies. ${ }^{78}$ The main reason for the lack of such studies is their complexity, duration, and cost due to the low incidence of adverse events. For an idea about the estimated sample size, a randomized, parallel-group study aimed at comparing the safety of agent $A$ versus agent $B$ with expected rates of allergic-type severe adverse reactions of $1 / 20,000$ and $1 / 100,000$, respectively, would require 350,000 patients per agent-that is, 700,000 patients for $85 \%$ statistical power by using 2 group Fisher exact tests with an $\alpha$ of .05 and a 2 -sided significance level. Such a sample size would be required for a 5 -fold difference in the rate of adverse reactions. If you assume that agent $\mathrm{A}$ has a reaction rate of $1 / 50,000$ and agent $B$ has a reaction rate of $1 / 100,000$, the number of patients per group for the same $85 \%$ power would rise to $2,890,000$.

Several reports have claimed to show differences across GBCAs. ${ }^{65,66}$ However, these reports have largely been retrospective surveys or observational studies with major methodologic flaws, such as reliance on the accuracy of historic recordkeeping by busy health care providers, lack of randomization, presence of unbalanced study groups, possible selection bias, potential for confounding data, and inappropriate analysis. In contrast, more reliable data are derived from prospective development studies. Unfortunately, most development studies are noncomparative and, when comparative, are typically too small to allow robust and unequivocal conclusions. ${ }^{78}$

\section{DISCUSSION}

\section{Morphologic Imaging}

One major conclusion to be drawn from intraindividual crossover studies performed to compare GBCAs at approved doses ( 0.1 $\mathrm{mmol} / \mathrm{kg}$ body weight) for morphologic imaging of brain lesions (mostly tumors) is that $r 1$-relaxivity is the primary determining 
factor for contrast efficacy. Thus, large-scale, well-controlled, prospective clinical studies have found no differences between GBCAs with similar $r 1$ values (Magnevist versus ProHance, ${ }^{26} \mathrm{Ga}-$ davist versus ProHance, ${ }^{31}$ ), while differences have been observed between GBCAs with higher r1-relaxivity versus GBCAs with "standard" r1-relaxivity (Gadavist versus Dotarem ${ }^{32}$; MultiHance versus Dotarem, Magnevist, Omniscan, Gadavist ${ }^{33-41}$ ). Moreover, the studies performed so far suggest that the magnitude of the superiority reflects the magnitude of the difference in $r 1$-relaxivity. Thus, the difference in percentage lesion enhancement of approximately $9 \%$ for Gadavist compared with Dota$\mathrm{rem}^{32}$ reflects only a slightly greater $r$-relaxivity of Gadavist compared with Dotarem, while the larger differences of 22\%-27\% for MultiHance compared with Gadavist ${ }^{41}$ and Magnevist, ${ }^{37}$ respectively, reflect the larger differences in $r 1$-relaxivity between these agents. Notably, small differences in percentage lesion enhancement have not been demonstrated to add significant clinical efficacy, ${ }^{31,32}$ while larger differences have been shown to result in significantly superior depiction of tumor morphologic features, lesion extent, and border delineation. ${ }^{33-41}$ The previously cited studies also support our earlier hypothesis that in steady-state postcontrast T1-weighted imaging typically used for conventional CNS imaging, in which postcontrast acquisition times are delayed at least 2-5 minutes following contrast agent injection, the administered Gd concentration of the agent does not play a significant clinical role when the different GBCAs are delivered at equal FDA-approved doses.

\section{Perfusion Imaging}

The magnitude of signal drop in DSC perfusion imaging is largely, if not solely, dependent on the $r 2^{*}$ value of the GBCA together with the local concentration of Gd. The $r 1$ and $r 2$ values of GBCAs play no detectable role in DSC perfusion. Thus, at least theoretically, the higher concentration of Gadavist should be advantageous for perfusion imaging compared with standard-concentration GBCAs when administered at equivalent doses at a fixed injection rate. Hence, while a $75-\mathrm{kg}$ patient administered a standard Gadavist dose of $0.1 \mathrm{mmol} / \mathrm{kg}$ body weight at a rate of $5 \mathrm{~mL} / \mathrm{s}$ would receive a total volume of $7.5 \mathrm{~mL}$ in 1.5 seconds, the same patient administered an equivalent gadolinium dose of standard concentration GBCA at the same rate would receive a total volume of $15 \mathrm{~mL}$ during 3 seconds. In this setting the possibility of injecting an equivalent number of $\mathrm{Gd}$ molecules in a shorter time, giving a sharper contrast bolus, might be expected to result in more Gd molecules in the tissue of interest during image acquisition and thus a greater susceptibility effect, as suggested by Giesel et al. ${ }^{44}$ However, the experimental results of Thilmann et $\mathrm{al}^{46}$ and Essig et $\mathrm{al}^{45}$ revealed no significant differences for similar injected doses of Gadavist and MultiHance (identical signal intensity/time curves obtained for single-dose examinations with both GBCAs). These findings suggest that bolus dispersion (ie, physiologic widening [dilution] of the contrast bolus during the first pass through the lungs and heart) leads to normalization of the resulting bolus shape to a point where the local intravascular concentration of Gd is similar for injections of equal total dose. Dilution of the contrast bolus in this manner appears to obviate any benefit of higher Gd concentration at the short injection times (typically
$<5$ seconds) required for perfusion imaging. ${ }^{46}$ In this case, the susceptibility perfusion effect is determined primarily by the administered GBCA dose, rather than by administered GBCA molarity. ${ }^{46}$ There is support for this possibility in a study by van Osch et al, ${ }^{80}$ which reported the altered results of perfusion measurements obtained when varying parameters, including injection rate, injection volume, and injection time, even when using the same total Gd dose.

\section{Contrast-Enhanced MR Angiography}

When injected under comparable conditions (ie, with identical bolus geometry), the greater $r$-relaxivity of MultiHance has been shown to be beneficial for CE-MRA of the supra-aortic arteries, giving superior quantitative and qualitative enhancement when administered at an equivalent dose to Magnevist ${ }^{51}$ and equivalent enhancement and diagnostic performance, when a single dose of MultiHance is compared with a double dose of Magnevist. ${ }^{50}$ These findings in the supra-aortic arteries are supported by the findings of numerous intraindividual crossover studies in other vascular territories. ${ }^{81-86}$

It is reasonable to conclude that increased GBCA concentration (as seen in Gadavist) and significant increases in $r$-relaxivities (to the magnitude seen with MultiHance) would each be beneficial for increased vascular signal intensity for CE-MRA, though the benefit obtained from higher concentrations may be accompanied by a concomitant shortening of the optimal image-acquisition timing window when both agents are injected at similar rates. Attempting to increase the likelihood of successfully matching the timing window of the lowest order phase-encoding steps with the first passage of the contrast agent by slower injection rates or by dilution of the administered agent may help offset any such timing problems but may simultaneously serve to negate any potential benefit that the higher concentration agent might have introduced.

\section{CONCLUSIONS}

The use of GBCAs in neurorardiologic applications has revolutionized the field since their introduction 25 years ago. Clinical applications in steady-state parenchymal imaging, MR angiography, and perfusion are developing rapidly. Radiologists should be aware in detail of the characteristics of the various agents including relaxivity, concentration, and chelate stability along with how these affect diagnostic efficacy and patient safety. In this review, we have attempted to present these in a manner that will, hopefully, enable the physician user to optimize agent selection and imaging parameters to achieve the best results for any given clinical situation.

Disclosures: Emanuel Kanal—UNRELATED: Consultancy: Guerbet, Comments: prior to their having a GBCA approved by the FDA, Grants/Grants Pending: Bracco Diagnostics, ${ }^{*}$ Comments: unrestricted grant funding to support the following project: "In Vitro Serial Dilution Study to Measure the Effects of Comparative Relaxivities among GBCA." Kenneth Maravilla_UNRELATED: Consultancy: Bracco, Guerbet, Grants/ Grants Pending: Bracco, Bayer, Guerbet, Payment for Lectures (including service on Speakers Bureaus): Bracco, Guerbet. Howard A. Rowley-RELATED: Grant: Bayer,* Bracco, ${ }^{*}$ Guerbet, ${ }^{*}$ GE Healthcare, ${ }^{*}$ Comments: clinical trials and research support, Consulting Fee or Honorarium: Bayer, Bracco, Guerbet, GE Healthcare, Comments: consulting fees related to contrast or contrast applications, Support for Travel to Meetings for the Study or Other Purposes: Bayer, Bracco, Guerbet, GE Healthcare, Comments: travel reimbursement related to consulting, UNRELATED: Consultancy: 
Gore, Eli Lilly, Genentech, Abbott Labs, Lundbeck, Paion, Forest Labs, National Institutes of Health, Comments: clinical trial safety monitoring boards, consulting for protocols which incorporated contrast use in some cases, Payment for Lectures (including service on Speakers Bureaus): Bracco, GE Healthcare, Continuing Medical Education organizations, Patents (planned, pending or issued): GE Healthcare, ${ }^{\star} \mathrm{Com}$ ments: MRI pulse sequence, joint patent pending, Royalties: Wisconsin Alumni Research Foundation, Comments: CT patent that incorporates contrast. *Money paid to the institution.

\section{REFERENCES}

1. Dotarem product label. http://www.accessdata.fda.gov/drugsatfda_ docs/label/2013/204781s000lbl.pdf. Accessed May 10, 2013

2. Gadavist product label. http://www.accessdata.fda.gov/drugsatfda_ docs/label/2011/201277s000lbl.pdf. Accessed May 10, 2013

3. Magnevist product label. http://www.accessdata.fda.gov/drugsatfda docs/label/2012/019596s051lbl.pdf. Accessed May 10, 2013

4. MultiHance product label. http://www.accessdata.fda.gov/drugs atfda_docs/label/2010/021357s009lbl.pdf. Accessed May 10, 2013

5. Omniscan product label. http://www.accessdata.fda.gov/drugs atfda_docs/label/2010/020123s037lbl.pdf. Accessed May 10, 2013

6. OptiMARK product label. http://www.accessdata.fda.gov/drugs atfda_docs/label/2010/020937s016lbl.pdf. Accessed 10 May 10, 2013

7. ProHance product label. http://www.accessdata.fda.gov/drugs atfda_docs/label/2010/020131s024lbl.pdf. Accessed May 10, 2013

8. Ablavar product label. http://www.accessdata.fda.gov/drugs atfda_docs/label/2010/021711s003lbl.pdf. Accessed May 10, 2013

9. Eovist product label. http://www.accessdata.fda.gov/drugsatfda_ docs/label/2010/022090s004lbl.pdf. Accessed May 10, 2013

10. Bellin MF, Van Der Molen AJ. Extracellular gadolinium-based contrast media: an overview. Eur J Radiol 2008;66:160-67

11. Rohrer M, Bauer H, Mintorovitch J, et al. Comparison of magnetic properties of MRI contrast media solutions at different magnetic field strengths. Invest Radiol 2005;40:715-24

12. Cavagna FM, Maggioni F, Castelli PM, et al. Gadolinium chelates with weak binding to serum proteins: a new class of high-efficiency, general purpose contrast agents for magnetic resonance imaging. Invest Radiol 1997;32:780-96

13. Giesel FL, von Tengg-Kobligk H, Wilkinson ID, et al. Influence of human serum albumin on longitudinal and transverse relaxation rates ( $\mathrm{R} 1$ and $\mathrm{R} 2)$ of magnetic resonance contrast agents. Invest $R a$ diol 2006;41:222-28

14. Pintaske J, Martirosian $\mathrm{P}, \mathrm{Graf} \mathrm{H}$, et al. Relaxivity of gadopentetate dimeglumine (Magnevist), gadobutrol (Gadavist), and gadobenate dimeglumine (MultiHance) in human blood plasma at $0.2,1.5$, and 3 Tesla. Invest Radiol 2006;41:213-21, Erratum in Invest Radiol 2006;41:859

15. Laurent S, Elst LV, Muller RN. Comparative study of the physicochemical properties of six clinical low molecular weight gadolinium contrast agents. Contrast Media Mol Imaging 2006;1:128-37

16. Yuh WT, Fisher DJ, Engelken JD, et al. MR evaluation of CNS tumors: dose comparison study with gadopentetate dimeglumine and gadoteridol. Radiology 1991;180:485-91

17. Myhr G, Rinck PA, Børseth A. Gadodiamide injection and gadopentetate dimeglumine: a double-blind study in MR imaging of the CNS. Acta Radiol 1992;33:405-09

18. Balériaux D, Matos C, De Greef D. Gadodiamide injection as a contrast medium for MRI of the central nervous system: a comparison with gadolinium-DOTA. Neuroradiology 1993;35:490-94

19. Valk J, Algra PR, Hazenberg CJ, et al. A double-blind, comparative study of gadodiamide injection and gadopentetate dimeglumine in MRI of the central nervous system. Neuroradiology 1993;35:173-77

20. Brugières $P$, Gaston $A$, Degryse $H R$, et al. Randomised double blind trial of the safety and efficacy of two gadolinium complexes (GdDTPA and Gd-DOTA). Neuroradiology 1994;36:27-30

21. Akeson P, Jonsson E, Haugen I, et al. Contrast-enhanced MRI of the central nervous system: comparison between gadodiamide injection and gadolinium-DTPA. Neuroradiology 1995;37:229-33

22. Oudkerk M, Sijens PE, van Beek EJ, et al. Safety and efficacy of Dotarem (Gd-DOTA) versus Magnevist (Gd-DTPA) in magnetic reso- nance imaging of the central nervous system. Invest Radiol 1995;30:75-78

23. Grossman RI, Rubin DI, Hunter G, et al. Magnetic resonance imaging in patients with central nervous system pathology: q comparison of OptiMARK (Gd-DTPA-BMEA) and Magnevist (Gd-DTPA). Invest Radiol 2000;35:412-19

24. Runge VM, Armstrong MR, Barr RG, et al. A clinical comparison of the safety and efficacy of MultiHance (gadobenate dimeglumine) and Omniscan (gadodiamide) in magnetic resonance imaging in patients with central nervous system pathology. Invest Radiol 2001;36:65-71

25. Runge VM, Parker JR, Donovan M. Double-blind, efficacy evaluation of gadobenate dimeglumine, a gadolinium chelate with enhanced relaxivity, in malignant lesions of the brain. Invest Radiol 2002;37:269-80

26. Greco A, Parker JR, Ratcliffe CG, et al. Phase III, randomized, double blind, crossover comparison of gadoteridol and gadopentetate dimeglumine in magnetic resonance imaging of patients with intracranial lesions. Australas Radiol 2001;45:457-63

27. Anzalone N, Gerevini S, Scotti R, et al. Detection of cerebral metastases on magnetic resonance imaging: intraindividual comparison of gadobutrol with gadopentetate dimeglumine. Acta Radiol 2009;50:933-40

28. Kim ES, Chang JH, Choi HS, et al. Diagnostic yield of double-dose gadobutrol in the detection of brain metastasis: intraindividual comparison with double-dose gadopentetate dimeglumine. AJNR Am J Neuroradiol 2010;31:1055-58

29. Katakami N, Inaba Y, Sugata S, et al. Magnetic resonance evaluation of brain metastases from systemic malignances with two doses of gadobutrol 1.0M compared with gadoteridol: a multicenter, phase II/III study in patients with known or suspected brain metastases. Invest Radiol 2011;46:411-18

30. Koenig M, Schulte-Altedorneburg G, Piontek M, et al. Intra-individual, randomised comparison of the MRI contrast agents gadobutrol versus gadoteridol in patients with primary and secondary brain tumours, evaluated in a blinded read. Eur Radiol 2013;23:3287-95

31. Center for Drug Evaluation and Research. FDA Advisory Committee Briefing Document. New Drug Application 201-277. http:// www.fda.gov/downloads/drugs/developmentapprovalprocess/ developmentresources/ucm255169.pdf. Accessed February 6, 2014

32. Anzalone N, Scarabino T, Venturi C, et al. Cerebral neoplastic enhancing lesions: multicenter, randomized, crossover intraindividual comparison between gadobutrol $(1.0 \mathrm{M})$ and gadoterate meglumine $(0.5 \mathrm{M})$ at $0.1 \mathrm{mmol} \mathrm{Gd} / \mathrm{kg}$ body weight in a clinical setting. Eur J Radiol 2013;82:139-45

33. Colosimo C, Ruscalleda J, Korves M, et al. Detection of intracranial metastases: a multicenter, intrapatient comparison of gadobenate dimeglumine-enhanced MRI with routinely used contrast agents at equal dosage. Invest Radiol 2001;36:72-81

34. Knopp MV, Runge VM, Essig M, et al. Primary and secondary brain tumors at MR imaging: bicentric intraindividual crossover comparison of gadobenate dimeglumine and gadopentetate dimeglumine. Radiology 2004;230:55-64

35. Colosimo C, Knopp MV, Barreau X, et al. A comparison of GdBOPTA and Gd-DOTA for contrast-enhanced MRI of intracranial tumours. Neuroradiology 2004;46:655-65

36. Essig M, Tartaro A, Tartaglione T, et al. Enhancing lesions of the brain: intra-individual crossover comparison of contrast enhancement after gadobenate dimeglumine versus established gadolinium comparators. Academic Radiology 2006;13:744-51

37. Maravilla KR, Maldjian JA, Schmalfuss IM, et al. Contrast enhancement of central nervous system lesions: multicenter intraindividual crossover comparative study of two MR contrast agents. $R a$ diology 2006;240:389-400

38. Kuhn MJ, Picozzi P, Maldjian JA, et al. Evaluation of intraaxial enhancing brain tumors on magnetic resonance imaging: intraindividual crossover comparison of gadobenate dimeglumine and 
gadopentetate dimeglumine for visualization and assessment, and implications for surgical intervention. J Neurosurg 2007;106:557-66

39. Rowley HA, Scialfa G, Gao PY, et al. Contrast-enhanced MR imaging of brain lesions: a large-scale intraindividual crossover comparison of gadobenate dimeglumine versus gadodiamide. AJNR Am J Neuroradiol 2008;29:1684-91

40. Rumboldt Z, Rowley HA, Steinberg F, et al. Multicenter, doubleblind, randomized, intra-individual crossover comparison of gadobenate dimeglumine and gadopentetate dimeglumine in MRI of brain tumors at 3 Tesla. J Magn Reson Imaging 2009;29:760-67

41. Seidl Z, Vymazal J, Mechl M, et al. Does higher gadolinium concentration play a role in the morphologic assessment of brain tumors? Results of a multicenter intraindividual crossover comparison of gadobutrol versus gadobenate dimeglumine (the MERIT Study). AJNR Am J Neuroradiol 2012;33:1050-58

42. Griffiths PD, Wilkinson ID, Wels T, et al. Brain MR perfusion imaging in humans. Acta Radiol 2001;42:555-59

43. Griffiths PD, Pandya H, Wilkinson ID, et al. Sequential dynamic gadolinium magnetic resonance perfusion-weighted imaging: effects on transit time and cerebral blood volume measurements. Acta Radiol 2006;47:1079-84

44. Giesel FL, Mehndiratta A, Risse F, et al. Intraindividual comparison between gadopentetate dimeglumine and gadobutrol for magnetic resonance perfusion in normal brain and intracranial tumors at 3 Tesla. Acta Radiol 2009;50:521-30

45. Essig M, Lodemann KP, Le-Huu M, et al. Intraindividual comparison of gadobenate dimeglumine and gadobutrol for cerebral magnetic resonance perfusion imaging at $1.5 \mathrm{~T}$. Invest Radiol 2006;41:256-63

46. Thilmann O, Larsson EM, Björkman-Burtscher IM, et al. Comparison of contrast agents with high molarity and with weak protein binding in cerebral perfusion imaging at $3 \mathrm{~T}$. J Magn Reson Imaging 2005;22:597-604

47. Wirestam R, Thilmann O, Knutsson L, et al. Comparison of quantitative dynamic susceptibility-contrast MRI perfusion estimates obtained using different contrast-agent administration schemes at 3T. Eur J Radiol 2010;75:e86-91

48. Beranek-Chiu J, Froehlich JM, Wentz KU, et al. Improved vessel delineation in keyhole time-resolved contrast-enhanced MR angiography using a gadolinium doped flush. J Magn Reson Imaging 2009;29:1147-53

49. Pediconi F, Fraioli F, Catalano C, et al. Gadobenate dimeglumine (Gd-BOPTA) vs gadopentetate dimeglumine (Gd-DTPA) for contrast-enhanced magnetic resonance angiography (MRA): improvement in intravascular signal intensity and contrast to noise ratio. Radiol Med 2003;106:87-93

50. Li Y, Li X, Li D, et al. Multicenter, intraindividual comparison of single-dose gadobenate dimeglumine and double-dose gadopentetate dimeglumine for MR angiography of the supra-aortic arteries (the Supra-Aortic Value Study). AJNR Am J Neuroradiol 2013;34:847-54

51. Bültmann E, Erb G, Kirchin MA, et al. Intra-individual crossover comparison of gadobenate dimeglumine and gadopentetate dimeglumine for contrast-enhanced MR angiography of the supraaortic vessels at 3 Tesla. Invest Radiol 2008;43:695-702

52. Kramer JH, Arnoldi E, François CJ, et al. Dynamic and static magnetic resonance angiography of the supra-aortic vessels at $3.0 \mathrm{~T}$ : intraindividual comparison of gadobutrol, gadobenate dimeglumine, and gadoterate meglumine at equimolar dose. Invest Radiol 2013;48:121-8

53. Pirovano G, Munley J, Schultz C, et al. Nephrogenic systemic fibrosis: a review of published cases and results from three prospective observational studies. Insights Imaging 2012;3(suppl 1):S293

54. Idée JM, Port M, Robic C, et al. Role of thermodynamic and kinetic parameters in gadolinium chelate stability. J Magn Reson Imaging 2009;30:1249-58

55. Bennett CL, Qureshi ZP, Sartor AO, et al. Gadolinium-induced nephrogenic systemic fibrosis: the rise and fall of an iatrogenic disease. Clin Kidney J 2012;5:82-88

56. Abujudeh HH, Kosaraju VK, Kaewlai R. Acute adverse reactions to gadopentetate dimeglumine and gadobenate dimeglumine: experience with 21,659 injections. AJR Am J Roentgentol 2010;194:430-34

57. American College of Radiology. Manual on Contrast Media. Version 9. 2013. http://www.acr.org. Accessed January 4, 2013

58. Bruder O, Schneider S, Nothnagel D, et al. Acute adverse reactions to gadolinium-based contrast agents in CMR: multicenter experience with 17,767 patients from the EuroCMR Registry. JACC Cardiovasc Imaging 2011;4:1171-76

59. Cochran ST, Bomyea K, Sayre JW. Trends in adverse events after IV administration of contrast media. AJR Am J Roentgenol 2001;176:1385-88

60. Forsting M, Palkowitsch P. Prevalence of acute adverse reactions to gadobutrol: a highly concentrated macrocyclic gadolinium chelate: review of 14,299 patients from observational trials. Eur J Radiol 2010;74:e186-92

61. Murphy KP, Szopinski KT, Cohal RH, et al. Occurrence of adverse reactions to gadolinium-based contrast material and management of patients at increased risk: a survey of the American Society of Neuroradiology Fellowship Directors. Acad Radiol 1999;6:656-64

62. Li A, Wong CS, Wong MK, et al. Acute adverse reactions to magnetic resonance contrast media: gadolinium chelates. $\mathrm{Br} J$ Radiol 2006;79:368-71

63. Dillman JR, Ellis JH, Cohan RH, et al. Frequency and severity of acute allergic-like reactions to gadolinium-containing I.V. contrast media in children and adults. $A J R A m \quad J$ Roentgenol 2007;189:1533-38

64. Hunt CH, Hartman RP, Hesley GK. Frequency and severity of adverse effects of iodinated and gadolinium contrast materials: retrospective review of 456,930 doses. AJR Am J Roentgenol 2009; 193:1124-27

65. Prince MR, Zhang H, Zou Z, et al. Incidence of immediate gadolinium contrast media reactions. $A J R A m$ J Roentgenol 2011;196:W138-43

66. Jung JW, Kang HR, Kim MH, et al. Immediate hypersensitivity reaction to gadolinium-based MR contrast media. Radiology 2012;264:414-22

67. Vervloet D, Durham S. Adverse reactions to drugs. $B M J$ 1998;316:1511-14

68. Hasdenteufel F, Luyasu S, Renaudin JM, et al. Anaphylactic shock after first exposure to gadoterate meglumine: two case reports documented by positive allergy assessment. J Allergy Clin Immunol 2008; $121: 527-28$

69. Schiavino D, Murzilli F, Del Ninno M, et al. Demonstration of an IgE-mediated immunological pathogenesis of a severe reaction to gadopentetate dimeglumine. J Invest Allergol Clin Immunol 2003;13:140-42

70. European Society on Urogenital Radiology (ESUR). ESUR Guidelines on Contrast Media. Version 8.1. http://www.esur.org/esur-guidelines/. Accessed January 4, 2013

71. United States of America ex rel. Lynch vs. Imagimed LLC, et al. (N.D. N.Y.); as reported on http://www.justice.gov/opa/pr/2013/ August/13-civ-958.html. Accessed September 14, 2013

72. Runge V. Safety of approved MR contrast media for intravenous injection. J Magn Reson Imaging 2000;12:205-13

73. Bleicher AG, Kanal E. Assessment of adverse reaction rates to a newly approved MRI contrast agent: review of 23,553 administrations of gadobenate dimeglumine. AJR Am J Roentgenol 2008;191:W307-11

74. Shellock FG, Parker JR, Venetianer C, et al. Safety of gadobenate dimeglumine (MultiHance): summary of findings from clinical studies and postmarketing surveillance. Invest Radiol 2006;41:500-09

75. Shellock FG, Parker JR, Pirovano G, et al. Safety characteristics of gadobenate dimeglumine: clinical experience from intra- and interindividual comparison studies with gadopentetate dimeglu- 
mine. J Magn Reson Imaging 2006;24:1378-85, Erratum in J Magn Reson Imaging 2007;26:217

76. Weber JC. Epidemiology of adverse reactions to nonsteroidal antiinflammatory drug. In: Rainsford KD, Velo GP, eds. Side Effects of Antiinflammatory/Analgesic Drugs Advances in Inflammation Research. Vol. 6. New York: Raven Press; 1984:1-7

77. Lalli AF. Urographic contrast media reactions and anxiety. Radiology 1974;112:267-71

78. Semelka RC, Hernandes Mde A, Stallings CG, et al. Objective evaluation of acute adverse events and image quality of gadoliniumbased contrast agents (gadobutrol and gadobenate dimeglumine) by blinded evaluation: pilot study. Magn Reson Imaging 2013;31:96-101

79. Davenport MS, Dillman JR, Cohan RH, et al. Effect of abrupt substitution of gadobenate dimeglumine for gadopentetate dimeglumine on rate of allergic-like reactions. Radiology 2013;266:773-82

80. van Osch MJ, Vonken EJ, Wu O, et al. Model of the human vasculature for studying the influence of contrast injection speed on cerebral perfusion MRI. Magn Reson Med 2003;50:614-22

81. Prokop M, Schneider G, Vanzulli A, et al. Contrast-enhanced MR angiography of the renal arteries: blinded multicenter crossover comparison of gadobenate dimeglumine and gadopentetate dimeglumine. Radiology 2005;234:399-408
82. Gerretsen SC, le Maire TF, Miller S, et al. Multicenter, double-blind, randomized, intraindividual crossover comparison of gadobenate dimeglumine and gadopentetate dimeglumine for MR angiography of peripheral arteries. Radiology 2010;255:988-1000

83. Knopp MV, Giesel FL, von Tengg-Kobligk H, et al. Contrast-enhanced MR angiography of the run-off vasculature: intraindividual comparison of gadobenate dimeglumine with gadopentetate dimeglumine. J Magn Reson Imaging 2003;17:694-702

84. Stein PD, Chenevert TL, Fowler SE, et al. Gadolinium-enhanced magnetic resonance angiography for pulmonary embolism: a multicenter prospective study (PIOPED III). Ann Intern Med 2010;152: 434-43, W142-43

85. Woodard PK, Chenevert TL, Sostman HD, et al. Signal quality of single dose gadobenate dimeglumine pulmonary MRA examinations exceeds quality of MRA performed with double dose gadopentetate dimeglumine. Int $J$ Cardiovasc Imaging 2012;28:295-301

86. Wang J, Yan F, Liu J, et al. Multicenter, intra-individual comparison of single dose gadobenate dimeglumine and double dose gadopentetate dimeglumine for MR angiography of the peripheral arteries (the peripheral VALUE study). J Magn Reson Imaging 2013;38:926-37 